\title{
Fractal Study on the Failure Evolution of Concrete Material with Single Flaw Based on DIP Technique
}

\author{
Lulin Zheng $\mathbb{D}^{1,3}$ Hao Liu $\mathbb{D}^{1,2}$ Yujun Zuo ${ }^{1},{ }^{1,2}$ Quanping Zhang, ${ }^{4}$ Wei Lin, ${ }^{5}$ Qing Qiu, ${ }^{1}$ \\ Xiaorong Liu, ${ }^{1}$ and Ziqi Liu ${ }^{1}$ \\ ${ }^{1}$ College of Mining, Guizhou University, Guiyang, Guizhou 550025, China \\ ${ }^{2}$ College of Resource and Environmental Engineering, Guizhou University, Guiyang, Guizhou 550025, China \\ ${ }^{3}$ School of Architecture and Civil Engineering, Xiamen University, Xiamen, Fujian 361005, China \\ ${ }^{4}$ School of Earth Sciences and Resources, China University of Geosciences (Beijing), Beijing 100083, China \\ ${ }^{5}$ Faculty of Engineering, China University of Geosciences, Wuhan, Hubei 430074, China \\ Correspondence should be addressed to Hao Liu; 907529196@qq.com
}

Received 22 December 2021; Accepted 17 January 2022; Published 4 February 2022

Academic Editor: Ren Fuqiang

Copyright ( $\odot 2022$ Lulin Zheng et al. This is an open access article distributed under the Creative Commons Attribution License, which permits unrestricted use, distribution, and reproduction in any medium, provided the original work is properly cited.

\begin{abstract}
Crack inclination and material heterogeneity have important effects on the meso-mechanical mechanism and macroscopic mechanical behavior of rock-like materials. In order to study the failure characteristics of shotcrete body during the process of using shotcrete bolt mesh support in the deep fractured rock mass of Lannigou Gold Mine, this paper combined the Digital Image Processing Technique (DIP) and RFPA2D (Rock Failure Process Analysis System) to establish a real meso-structure numerical model of concrete with different inclination angle cracks, simulating its crack propagation law and failure process, and studied the influence of crack geometry distribution and meso-heterogeneity on the effect of concrete structure. The findings reveal that the crack inclination angle has a substantial impact on concrete materials' compressive strength and elastic modulus, and both of them all show a nonlinear increase with the increase of crack angle; Because of the inhomogeneity of the materials, the inclination and propagation pathways of wing cracks are random, and the aggregate inhibits crack initiation and propagation. The wing crack's initiation position moves closer to the tip as the crack inclination angle increases, and the length gets shorter; Acoustic emission(AE) evolution characteristics are similar in samples with varying dip angles. In the early stages of loading, the AE energy is minimal, and increases rapidly when approaching the peak stress. The fractal dimension was used to describe the damage evolution process inside the material, and a damage variable index $(\omega)$ based on the fractal theory was proposed. The more the $\omega$, the greater the material's degree of degradation. The proposed index provided a new method for quantitative study of the damage evolution characteristics of rock-like materials. It has guiding significance for the research on the stability of wet shotcrete in the deep fractured rock mass of Lannigou Gold Mine.
\end{abstract}

\section{Introduction}

The Lannigou Gold Mine is located in Zhenfeng county, the Southwest of Guizhou province. The underground mining utilizes ramp development and layered upward cemented fill mining. In order to ensure the safety of mining operations, shotcrete anchor nets are used to reinforce the surrounding rock in almost all roadways in the mine. In particular, wet shotcrete is the most important process to reinforce the roadways, which is particularly effective in the weathered rock mass of the mine. As the mining depth increases, the deep ground stress increases. Meanwhile the surrounding rock is fragmented and the stability is poor due to the combined influence of high ground stress and geological structure. As a result, investigating the fracture characteristics and damage development process of wet shotcrete can provide some theoretical support for the concrete support of the Lannigou gold mine and other comparable mines.

As an artificial rock-like material, concrete contains different types and structures of media. They have different 
physical and mechanical properties, containing a large number of defects, and their responses to external forces are also very different[1-3]. The initiation and propagation behavior of cracks in rock-like materials is closely related to their internal structures[4-6], single crack angle and material heterogeneity jointly determine the macroscopic fracture characteristics and damage evolution characteristics of material. As a result, studying the fracture process and damage evolution characteristics of rock-like materials while taking into account the crack inclination angle and material non-uniformity, as well as revealing the mesoscopic damage evolution mechanism and macroscopic nonlinear mechanical behavior, is of great theoretical importance.

Scholars in the China and around the world have never stopped studying the initiation and propagation behavior of rock cracks and rock-like materials cracks around the crack inclination angle. Nolen-Hoeksema et al. conducted compression tests on marble and discovered that when the prefabricated crack is oblique, the initiation and propagation of the tip secondary cracks are asymmetrical[7]. Yang et al. study the influence of crack angle on the initiation behavior and compressive strength of sandstone through rock mechanics experiments[8]. Park et al. conducted rock mechanics experiments on rocks with cracks, and the study found that as the crack inclination angle increased, so did the initiation stress of the wing crack[9]. Manouchehrian et al. investigated the role of crack inclination in the initiation and propagation of rock cracks using numerical simulation methods[10]. Through numerical simulation, Zhang et al. investigated the impact of fracture angle on the initiation process of rock-like materials, finding that the crack inclination angle has a significant impact on crack initiation and propagation[11]. Liu et al. used a combination of physical tests and numerical simulations to investigate how crack inclination affects crack propagation and stress field evolution in rock-like materials[12]. Xu et al. used physical experiments to investigate the effect of fracture angle on the crack initiation behavior of rock-like materials under uniaxial compression, finding that the uniaxial compressive strength increased as the crack angle rose[13]. However, because the macroscopic damage of materials is closely related to the development and aggregation of internal micro-cracks, the evolution of material damage has aroused widespread concern of many scholars. Taylor et al. used the self-consistent approach to create a link between crack density and rock damage, and evaluated the completeness of fractures in the rock to reflect the damage degree[14]. Ahrens et al. linked the development of cracks in the medium with the change of the p-wave velocity, and used p-wave velocity to characterize the distribution characteristics of micro-cracks inside the rock mass, so as to evaluate the damage of surrounding rock according to the change of p-wave velocity[15]. Xie developed fractal-damage mechanics by applying fractal geometry theory to the analysis of rock fractures, opening up a new technique to examine rock damage quantitatively[16]. Gao has studied the mechanical behavior of coal and rock under various mining circumstances, from laboratory experiments to field testing[17]. Based on the fractal theory, Liu et al. compiled an algorithm pf rock mesoscale fracture box dimension based on digital images, proposed a calculation method of rock mesoscale fracture dimension, and carried out quantitative characterization of the failure mode and damage degree of jointed sandstone $[4,18]$.

The above research results are of great significance for revealing the crack propagation mechanism and damage evolution characteristics of materials with flaw at macroscale, but the macroscopic mechanical behavior of materials is determined by the heterogeneity and mesoscale behavior of materials. Therefore, the study on the fracture process and damage evolution characteristics of the material on the basis of considering the material inhomogeneity and flaw inclination will help reveal its crack propagation mechanism and macroscopic nonlinear mechanical behavior.

The true mesoscopic structure of varied angle cracks in concrete numerical model is established in this research using the Rock Failure Process Analysis System (RFPA2D) and Digital Image Processing technology, the influence of crack geometric distribution and meso-uniformity on the concrete structure effect is studied, and the fractal theory is applied to describe the damage evolution process of material inside. The damage variable index, based on fractal theory, is offered as a new tool for studying the damage evolution of rock-like materials quantitatively.

\section{Finite element method for rock-like material failure process}

2.1. Digital image representation of meso-structure. The main components of concrete are aggregate, cement mortar, and accompanied by a small amount of primary holes (Figure 1(a)). The three components have different colors, among which gray is mostly mortar, white is aggregate, and the rest are holes. Figure 1(c) is a CT slice obtained by scanning with high-resolution industrial CT (Figure 1(b)) at Tianjin Sanying Company, and the image pixels are 200x200 pixels, the actual size is $100 \mathrm{~mm} \times 100 \mathrm{~mm}$. It is difficult to build a relationship between the unified algorithm and the finite element approach because the medium in the original image (Figure 1(c)) is dark or bright in color. Therefore, image processing is required to identify the mesoscopic medium inside the concrete.

Through the differences of grayscale and color, digital image processing technology distinguishes the geometric shape and spatial distribution of the material's meso-component. The technology is utilized to establish the segmentation threshold for distinct media in the rock based on color and brightness differences. The value divides the distribution of each medium, and the non-uniformity characterization image of the material can be obtained $[5,19]$.The Figure 1 (c) is a 24-bit BMP bitmap, whose color change is not visible. As a result, multi-threshold segmentation is performed by examining the variation rule of the brightness I value in HIS (Hue refers to chromaticity, S refers to Saturation, and I refers to brightness) image processing color space $[4,18]$. The changing curve of the value of I on the AA' scan line in Figure 1(c) is shown in Figure 1(d). The material medium passed by the scan line is compared with 


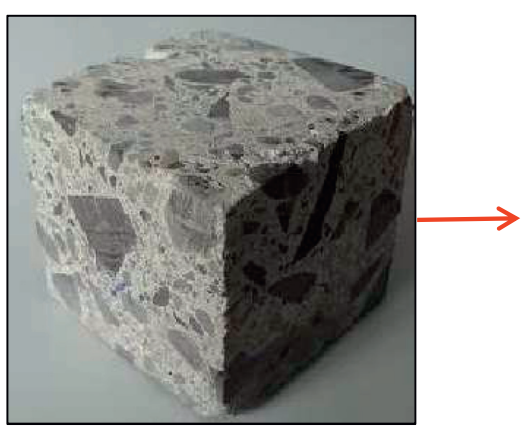

(a)

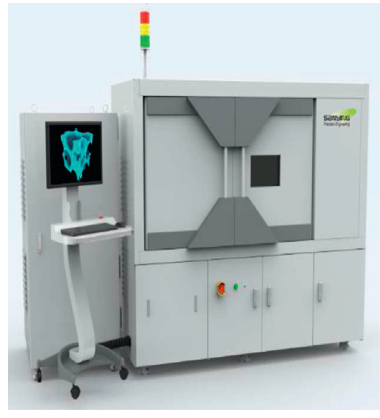

(b)

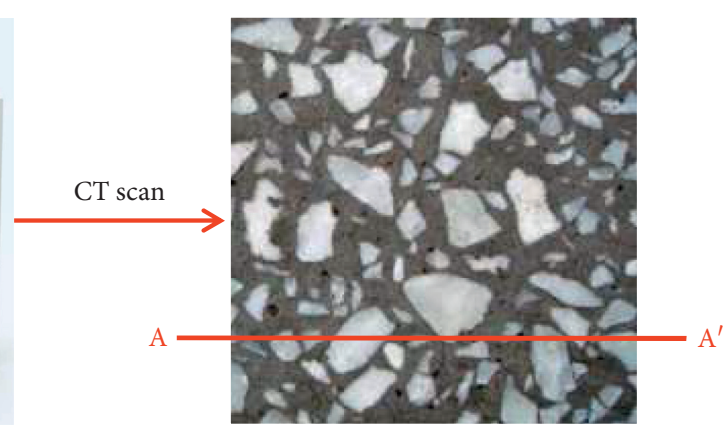

(c)

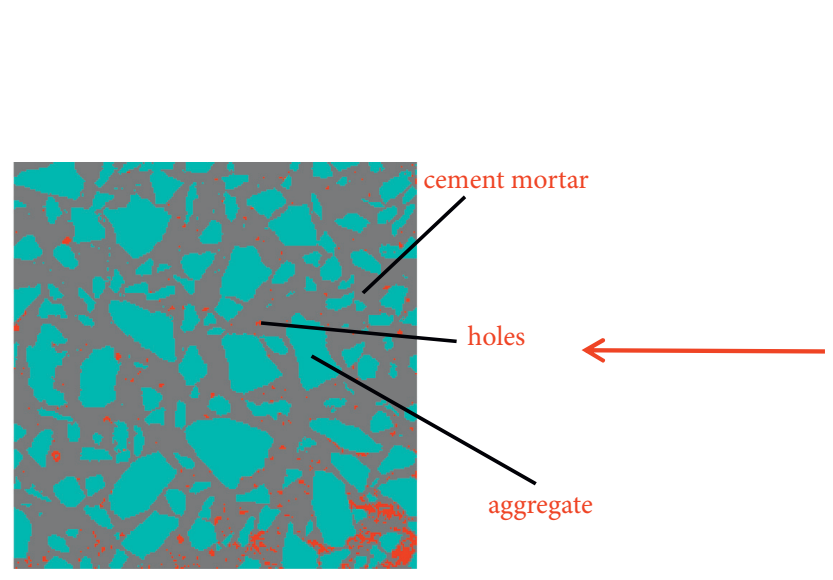

(e)

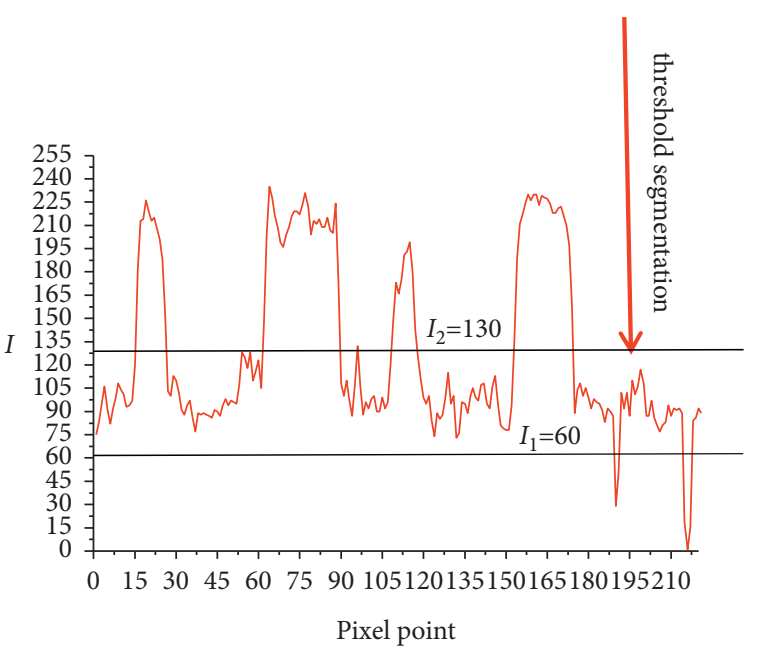

(d)

Figure 1: The process of concrete digital image characterization.

the change of the curve. Through repeated tests and adjustments in DIPS software, the ideal segmentation threshold is finally obtained, which is 60 and 130 respectively, and the characterization image can accurately reflect each mesoscopic component in Figure 1(c). Since the twodimensional CT slices are grayscale images, the value range of the matrix elements of the grayscale image is usually $[0,255]$, and 0 255 represents the range of color transition from pure black to pure white. Therefore, the I value is divided into three intervals: 0-60, 61-130, and 131-255. Each interval represents holes, mortar, and aggregates. That is, the internal mesoscopic medium of concrete is divided into 3 categories. Figure 1(e) is the meso-structure characterization image obtained after CT slice processing with DIPS software. Compared with Figure 1(c), the processed results basically reflect the real structure of concrete.

\subsection{Meso-structure transformation and numerical modeling.} The non-uniformity characterization of digital image processing technology must be constructed with a finite element mesh and then incorporated into the numerical model so that the influence of the material meso-structure may be considered in mechanical calculations and analyses. A digital image is a matrix of rectangular pixels, each of which is a small square with a certain width and height. As a result, each pixel in the finite element grid (Figure 2) can be considered a element, and the coordinates of its four corner points can be transformed into the appropriate actual position in vector space. The whole image can be converted into square finite element grid data and imported into RFPA2D to generate concrete numerical specimens. Detailed description of the conversion process and RFPA2D principle can be found in the literature[20,21]. The material properties of each material component are then allocated to the represented colors, and the non-uniformity coefficients of different components are input into the numerical model to create a numerical model that considers the material's genuine mesoscopic structure.

Given the non-uniformity of the material, we assume that the mechanical characteristics (elastic modulus, strength, etc.) of matrix elements obey the Weibull distribution function in the numerical calculation[22]:

$$
f(u)=\frac{m}{u_{0}}\left(\frac{u}{u_{0}}\right)^{m-1} \exp -\left(\frac{u}{u_{0}}\right)^{m},
$$

where, $u$ is the physical property parameter (elastic modulus, strength, etc.) of the material medium element; the average of the basic physical property characteristics is $u_{0}$; $m$ is the distribution function's property parameter that reflects the uniformity of the material medium; the smaller $m$, the more uneven the rock property. The statistical density of the physical attributes of the material constituents is denoted by 


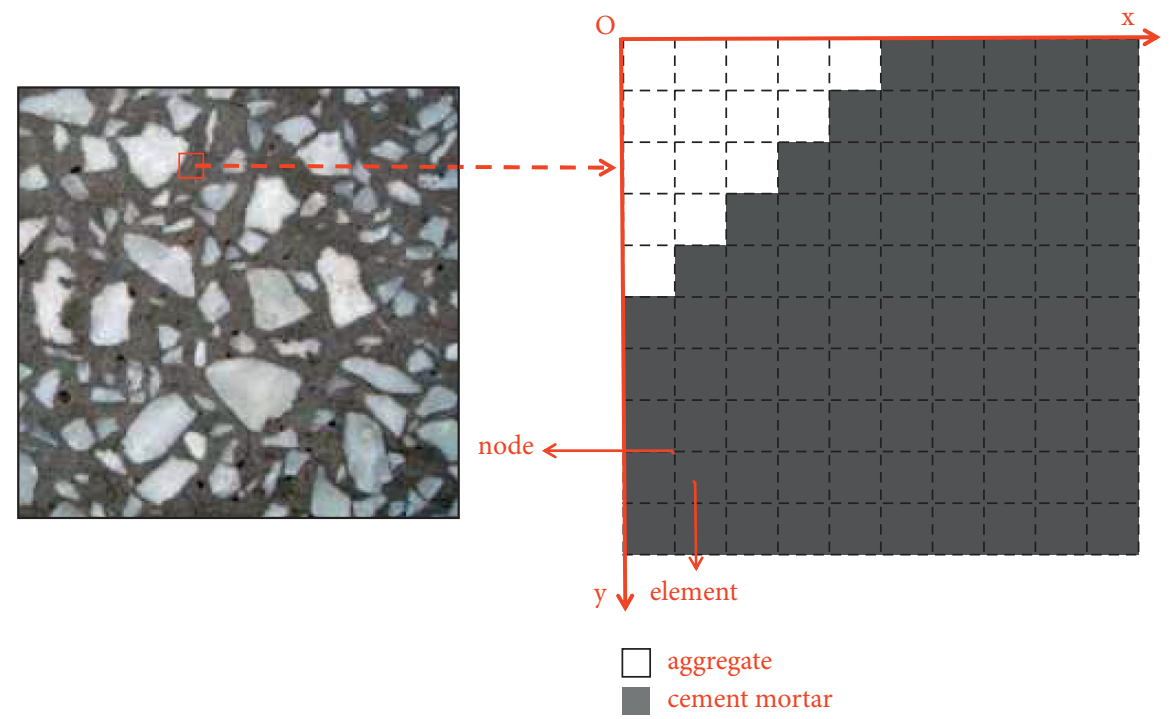

Figure 2: Concrete CT slice and finite element mesh transformation.

$f(u)$. The non-uniformity of aggregate, mortar, and primary cavity was taken into account in the model, and the mechanical characteristics of the meso-element were assigned using the Monte-Carlo approach[23, 24].

Table 1 shows the material properties of the meso-medium within the concrete, and the specimens are mechanically loaded by uniaxial compression. Figure 3 is a schematic diagram of the force on the numerical model, the entire process is controlled by a continuous displacement loading method, with plane stress analysis used to simulate the test. The starting uniaxial compression displacement value is $0.001 \mathrm{~mm}$, with a single step load increment of $0.002 \mathrm{~mm}$, a pressure ratio of 10 , and the specimen is loaded until it is destroyed. Considering the characteristics of concrete materials that are compressive and not tensile, this paper chooses the Mohr-Coulomb strength theory with tensile criterion[25] as the criterion for element failure. The numerical test was carried out in RFPA2D software[4, 5].

To investigate the impact of different inclination cracks on the mechanical properties of concrete materials, as well as the effect of microscopic non-uniformity caused by different crack geometrical distributions on the macroscopic failure of concrete, we prefabricated cracks with a length of $60 \mathrm{~mm}$ and a width of $5 \mathrm{~mm}$ in the middle of the sample, and anticlockwise every $15^{\circ}$ to obtain a digital image, a total of 5 digital image were acquired at last (as shown in Figure 4). The prefabricated crack dip angles $\alpha$ are $15^{\circ}, 30^{\circ}, 45^{\circ}, 60^{\circ}$ and $75^{\circ}$ respectively, where $\alpha$ is the prefabricated crack's included angle with respect to the horizontal direction. Furthermore, it is vital to ensure the same meso-structure of concrete specimens in order to analyze the fracture process and damage features of concrete with varying dip angles.

\section{Results and discussion}

3.1. Analysis of the influence of a single flaw on the mechanical properties of concrete. Figure 5 depicts the elastic modulus and internal primary stress distribution of concrete specimens when $\alpha=45^{\circ}$. The greater the brightness in the figure, the greater the stress value. Because of the variation in internal microstructure, the internal stress distribution of the specimen is uneven when compared to Figure 5(a). The stress concentration distribution at the tip of the prefabricated crack and the critical surface (weak surface) where the aggregate contacts the mortar is more significant. This demonstrates that the presence of cracks in concrete and material non-uniformity have a significant impact on stress distribution.

The compressive strength and elastic modulus of cracked concrete specimens with various inclination angles can be shown in Table 2. The elastic modulus and peak strength of various specimens are clearly anisotropic, as shown in Fig. 6, which increases non-linearly with the increase of the precrack inclination angle. The specimens' greatest strength is reached at $\alpha=75^{\circ}$, and the value is $143.84 \mathrm{MPa}$; while the minimum value is $93.71 \mathrm{MPa}$ when $\alpha=15^{\circ}$. This is due to the prefabricated cracks with different inclination angles in the specimen and the different mechanical properties of different components in the material, which lead to different mechanical responses under the same level of loading conditions, resulting in uneven stress distribution. This conclusion is in line with the findings of $\mathrm{Li}$ et al. [26] (Figure 6), indicating that the numerical simulation results are trustworthy. As shown in Figures 6 and 7, different stress concentrations are caused by the difference in the internal microstructure of the cracked concrete at different inclination angles when the specimen is loaded, which induces damage and gradually breaks. The elastic modulus and compressive strength show a great difference, which caused by the different inclination angles of the cracks.

3.2. Analysis of crack propagation characteristics and mesofracture evolution of specimens. Many researchers have looked at the start and propagation of the original fracture point and discovered that it may be split into three types: 
TABLE 1: Concrete mesoscopic medium material parameters

\begin{tabular}{lcccc}
\hline Material & Elastic modulus/GPa & Poisson ratio & Compressive strength/MPa & Internal friction angle/(C $\left.{ }^{\circ}\right)$ \\
\hline Aggregate & 49.7 & 0.25 & 298.8 & 35 \\
Cement mortar & 24.8 & 0.18 & 149.6 & 30 \\
\hline
\end{tabular}

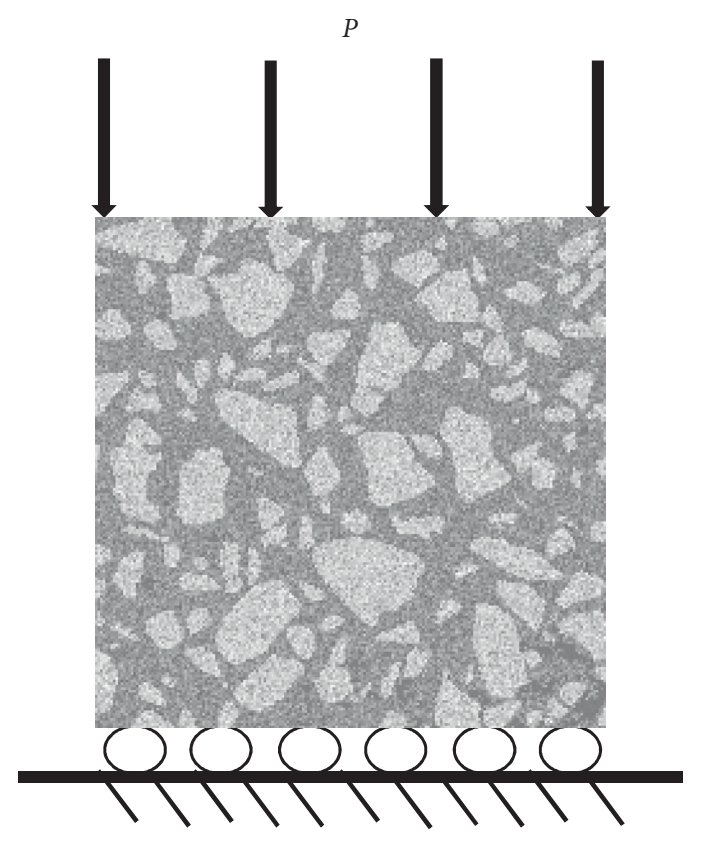

(a)

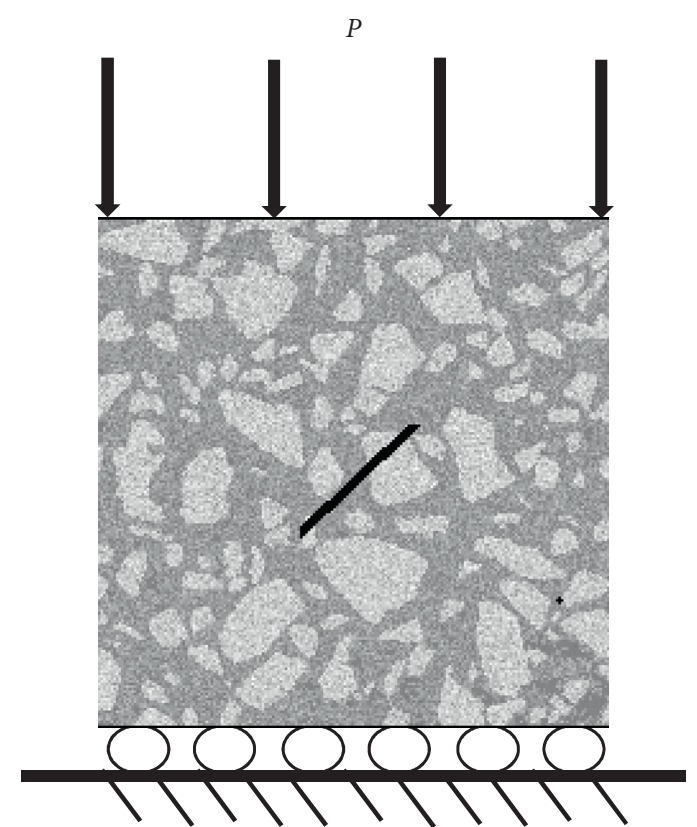

(b)

FIgURE 3: Schematic diagram of numerical model loading.

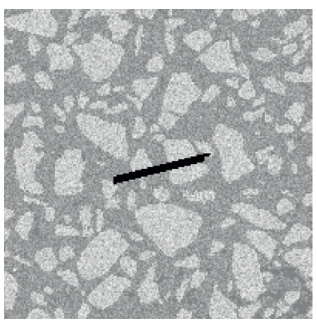

$\alpha=15^{\circ}$

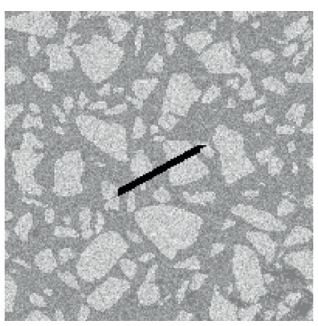

$\alpha=30^{\circ}$

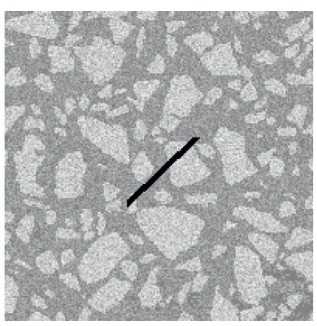

$\alpha=45^{\circ}$

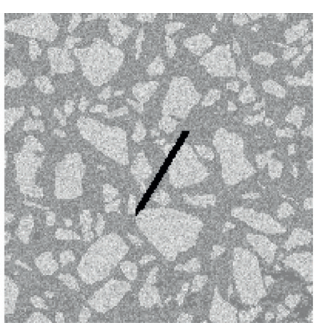

$\alpha=60^{\circ}$

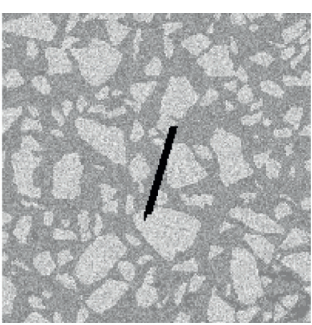

$\alpha=75^{\circ}$

Figure 4: Digital images of concrete materials with different inclination angles flaw.

Wing Crack (WC), Secondary Coplanar Crack (SCC) and Secondary Inclined Crack (SIC) [27, 28]. Figure 7 shows the evolution of acoustic emission and elastic modulus during the failure process of concrete with different inclination angles. In the acoustic emission evolution diagram, yellow indicates tensile failure, red indicates compression-shear failure, and black indicates damaged elements.

As can be seen from the Figure 7, When $\alpha=15^{\circ}$, the wing crack initiates and propagates along a specific distance from the tip of the prepared fracture, and the wing crack at the lower end propagates along the weak surface. The tensile stress determines the initiation and progression of the wing crack, and a secondary oblique crack is generated on the weak side of the lower right corner of the specimen due to the dominant tensile stress. With the increase of stress, the wing crack near the prefabricated crack's tip continued to spread until it penetrated, resulting in the specimen's tensile split failure; When $\alpha=30^{\circ}$, the wing crack is perpendicular to the prepared crack's tip and expands slowly; when axial stress increases, the upper wing crack penetrates the aggregate, resulting in transgranular cracks, while the lower wing crack continued to expand along the weak surface. As a result of the large accumulation of tensile failure, on the weak surface of the lower right corner of the specimen, a macroscopic secondary oblique crack developed, eventually leading to the splitting failure of the specimen; When $\alpha=45^{\circ}$, 


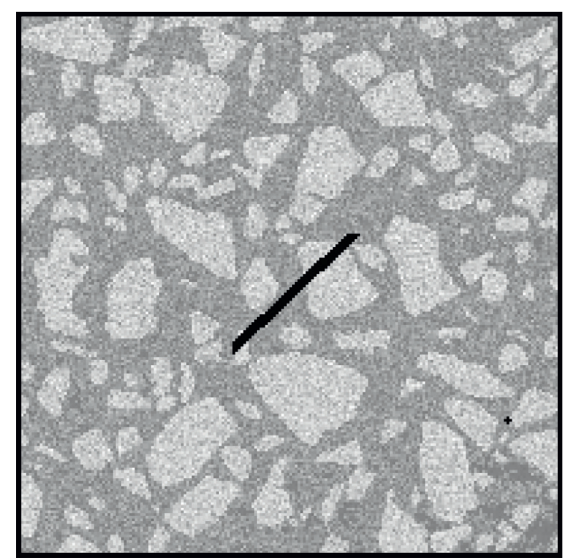

(a)

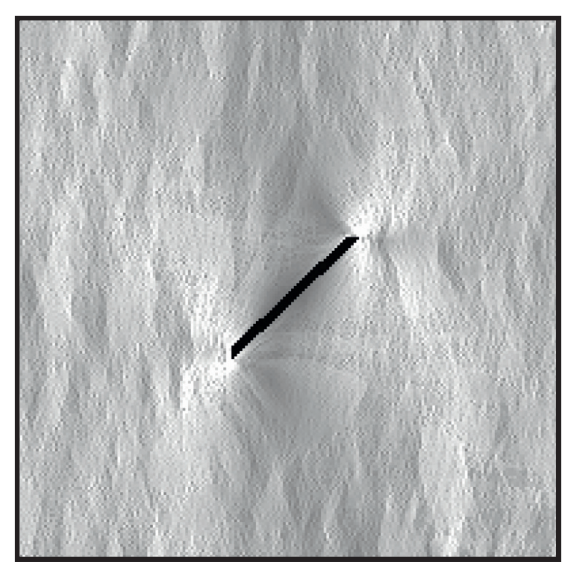

(b)

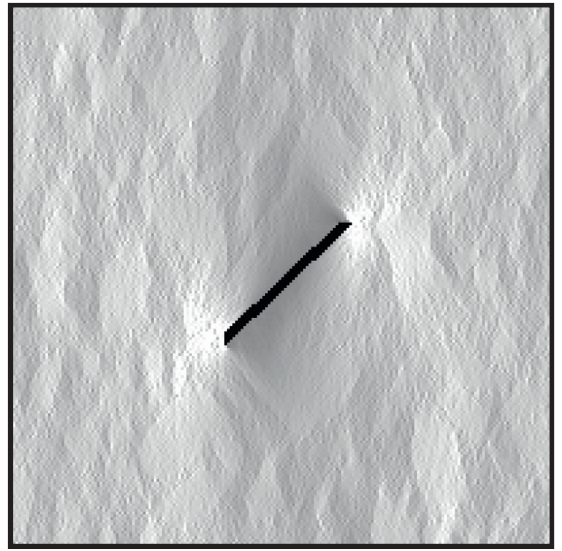

(d)

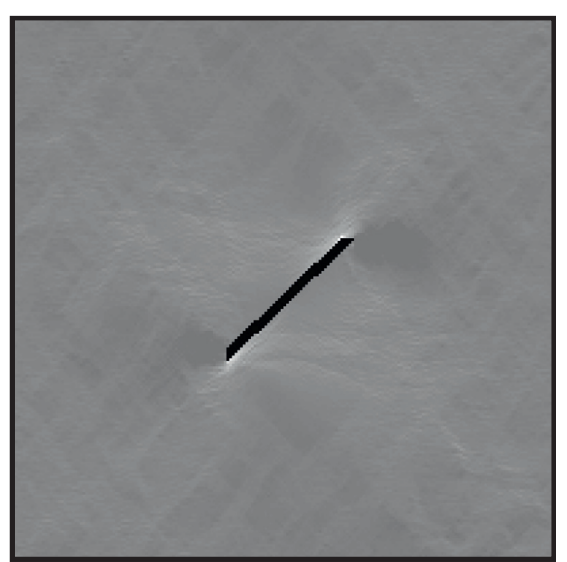

(c)

FIgURE 5: The specimen's elastic modulus and primary stress distribution diagram at the initial loading stage.

TABle 2: Concrete compressive strength and elastic modulus simulation results

\begin{tabular}{lcc}
\hline Inclination $/\left(^{\circ}\right)$ & Compressive strength(MPa) & $\begin{array}{c}\text { Elastic } \\
\text { modulus(GPa) }\end{array}$ \\
\hline$\alpha=15^{\circ}$ & 93.71 & 22.31 \\
$\alpha=30^{\circ}$ & 94.35 & 23.01 \\
$\alpha=45^{\circ}$ & 103.86 & 23.88 \\
$\alpha=60^{\circ}$ & 112.38 & 25.47 \\
$\alpha=75^{\circ}$ & 143.84 & 25.68 \\
\hline
\end{tabular}

the initiation and propagation of wing cracks are almost the same as when $\alpha=30^{\circ}$, but the failure process is relatively complicated, when the peak strength is approaching, a sudden initiation in the lower right part of the sample is caused by the large accumulation of tensile damage, the secondary coplanar cracks expanded rapidly, a secondary coplanar crack and a secondary oblique crack appeared in the upper left part of the sample. Eventually, all the cracks penetrated and the specimen was destroyed; All of the wing cracks initiated and expanded along the tip of the prefabricated crack when $\alpha=60^{\circ}$. As the continuous accumulation of axial stress, a large number of tensile failures occurred near the peak strength, resulting in the rapid initiation of a secondary oblique crack in the lower right part of the specimen, and a rapid initiation of the weak surface in the upper left part, coplanar cracks are generated, and the final grain penetration leads to macroscopic failure of the specimen; The tips of the prefabricated crack are located in the high-strength aggregate when $\alpha=75^{\circ}$, resulting in wing cracks at the tip of the prefabricated crack during the entire loading process, the expansion is slow, and when the peak strength is approaching, a large amount of tensile failure occurs on the weak surface of the lower right part and the upper end of the sample due to the accumulation of stress, the crack finally penetrates and the sample is unstable and damaged.

The components of tensile failure (yellow) dominate the acoustic emission development diagram, and the macroscopic shear bands generated by the specimen's failure are primarily related by the elements of tensile failure. In the process of concrete failure, the initiation and propagation of wing cracks have a certain randomness, with the increase of the inclination of the prefabricated cracks, the length of the wing cracks becomes shorter and the initiation position of the cracks keeps approaching the tip. When $\alpha=45^{\circ}$ and $\alpha=60^{\circ}$, the rupture process of the specimen is more complicated; When $15^{\circ} \leq \alpha \leq 45^{\circ}$, it is easier to initiate and propagate wing cracks, when $\alpha=60^{\circ}$ and $\alpha=75^{\circ}$, the initiation and propagation of wing cracks is problematic, and when the 


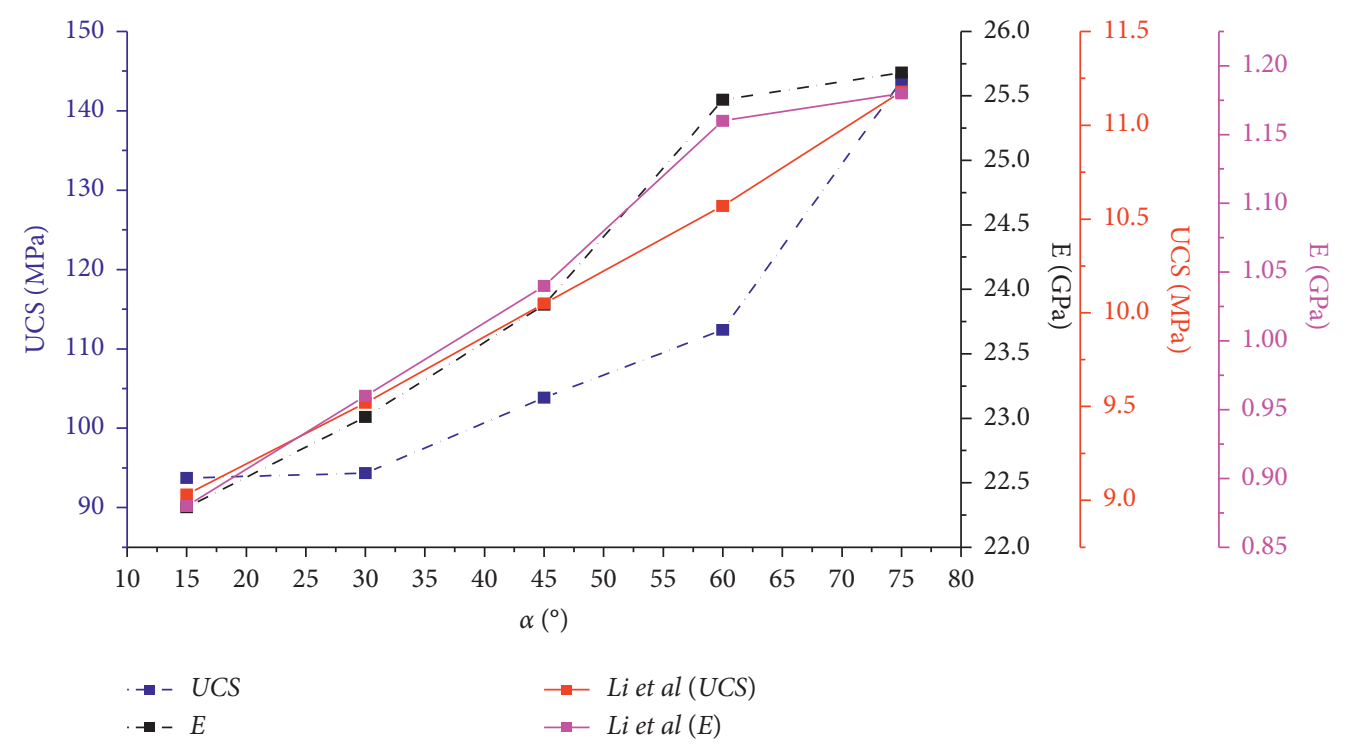

FIGURE 6: Cracked concrete peak strength and elastic modulus at various inclination degrees.

Elastic modulus evolution diagram of concrete fracture process

(a) $15^{\circ}$

(b) $30^{\circ}$

(c) $45^{\circ}$

(d) $60^{\circ}$

(e) $75^{\circ}$
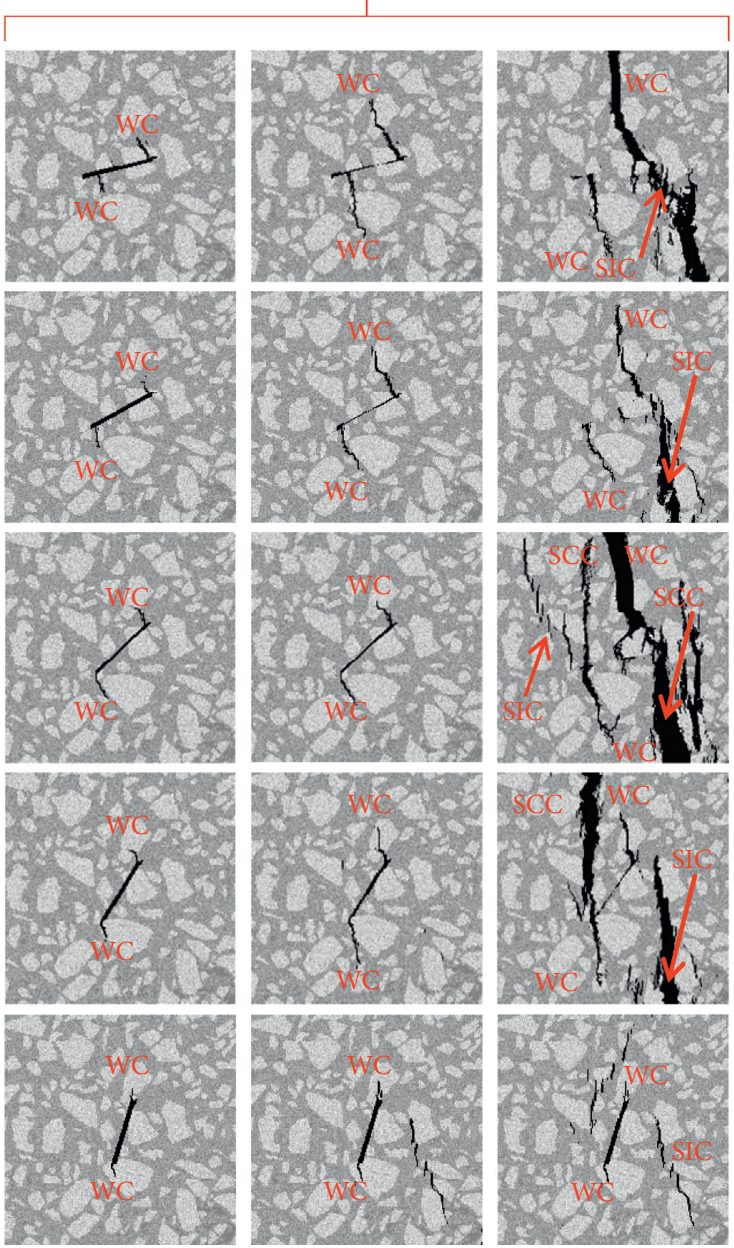

Acoustic emission evolution diagram of concrete fracture process
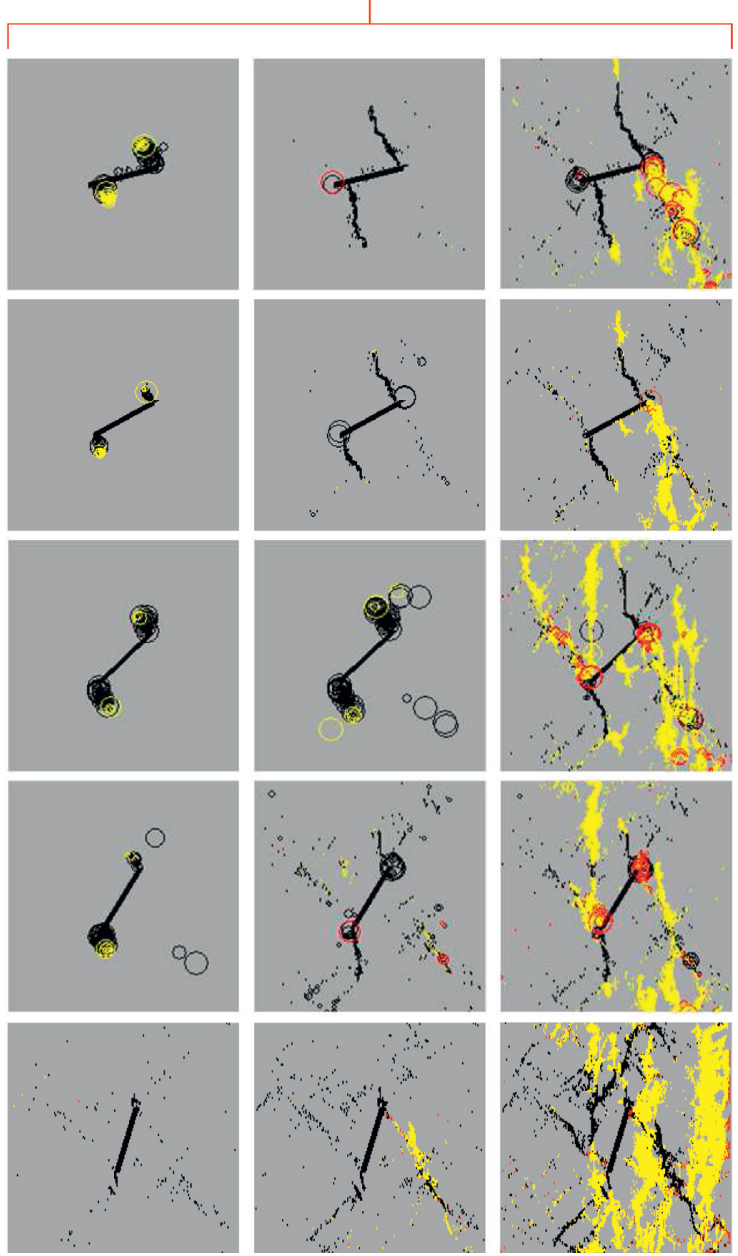

FIGURE 7: Failure process and acoustic emission evolution diagram of cracked concrete at different inclination angles. 
tension is near the peak stress, cracks begin to form and spread quickly, which will eventually lead to split failure. From the evolution diagram of elastic modulus, it can be seen that the initiation and propagation of cracks first spread along the weak plane due to the microscopic inhomogeneity. When the stress increases, the crack penetrates the aggregate to form transgranular cracks, which causes the crack to grow along the weak plane, with a regular expansion path, the specimen will eventually produce a tensile stress concentration area at the crack tip, which leads to crack penetration and tensile split failure. The shape of the inherent crack defects in concrete has an important influence on its failure mode. Furthermore, the aggregate acts as a barrier to the initiation and development of cracks.

\subsection{The law of energy change in the process of damage} evolution. Acoustic emission is an efficient instrument for studying the evolution process of internal rock damage since it relates to the elastic wave released by the quick release of local energy under load. In the RFPA2D numerical model, the method of stiffness degradation is used to deal with the failure elements, each failure element can be regarded as a micro-failure, and a element micro-failure is an acoustic emission event. The position of the acoustic emission is the center of the element and the magnitude of the acoustic emission energy, it is the energy released by the microfailure of the element, and the total number of damaged elements in each step can be used as the acoustic emission count at the current moment, and the cumulative number of damaged elements is the cumulative number of acoustic emissions. The accumulation of continuously damaged elements causes gradual damage to the entire material[20, 21]. The AE counts, cumulative AE counts, and stress changes of different inclination flaw samples with the loading step are shown in Figure 8.

Figure 8 shows that as the loading step is increased, the stress grows linearly until the peak strength is reached, at which point the stress drops significantly, while there is still some residual strength. The entire process can be divided into three stages: elastic, yield, and failure. There are less damage elements in the material at the start of the loading process, resulting the $\mathrm{AE}$ count and cumulative $\mathrm{AE}$ count are also less (paragraph 0 -I in Figure 8). As the continuous action of stress, there are more and more damage elements inside the material, a large number of damaged elements gather to form micro-cracks, the $\mathrm{AE}$ count reaches the maximum when the stress reaches the peak value, and the stored elastic energy is suddenly released, causing the crack to penetrate and the sample to be unstable and failure. During the loading process, as the stress continues to release more and more dissipated energy, the release of the dissipated energy of each sample has similar change characteristics. In the early stage, the AE energy is small, and it increases rapidly when it is close to the peak stress. The cracks grow slowly at the initial stage, and the cracks mainly grow and penetrate rapidly when they are close to the peak strength. The cumulative AE energy grows almost exponentially with the increase of the loading step before reaching the peak stress. Flat phase (0-I section), acceleration period (I-II section), and inflation period (II-III section) are the three stages of the growth process. The accumulated $\mathrm{AE}$ energy in the flat period mainly comes from the dissipated energy, after the acceleration period, the accelerated growth of the cracks in the material leads to the release of part of the elastic energy, and the accumulated AE energy increases rapidly. When the peak strength is reached, the internal cracks in the material penetrate and cause the sample macroscopic damage and the accumulated AE energy reaches its peak instantaneously.

\subsection{Fractal characteristics of damage evolution of concrete materials with flaw}

3.4.1. Fractal analysis of images using the box-counting method. Fractal theory can effectively describe the internal regularity of many irregular appearances in nature, and has shown its unique application prospects in many fields such as geotechnical engineering, biology, physics, and computer graphics $[4,16,18,28]$. Fractal dimension is one of the core contents of fractal theory, fractal dimension is used to replace the integer dimension of traditional European space, which can quantitatively characterize things with non-integer value dimension. While there are many ways to define the fractal dimension, the box-counting dimension can directly reflect the pixels occupied by the damaged element in the picture and the calculation is simpler than other methods. This paper chooses the box-counting dimension to test the concrete with flaw, such damage evolution characteristics are studied. The principle is that a square grid $(\delta \times \delta)$ is used to cover the damaged element in the image and the grid size is variable. Given the size of the box $\delta$, the number of boxes $N$ which required to cover the failure element can be calculated. Assuming that the number of boxes required to cover the destruction element with a square box of size $\delta_{i}$ in step i is $N_{i}\left(\delta_{i}\right)$, the size of the box in step $i+1$ is $\delta_{i}+1$, and the number of boxes required is $N_{i}+1\left(\delta_{i}+1\right)$. In the covering process, a set of $(\delta, N)$ data will be obtained, and the data will be fitted into a straight line in the logarithmic graph, and the slope of the straight line is equal to the boxcounting dimension $D$ of the set, which can be expressed as formula (2).

$$
D=-\lim _{\delta \longrightarrow 0} \frac{\lg N(\delta)}{\lg \delta} .
$$

Based on the above understanding and the principle of digital image storage, combined with the digital image analysis technology and Matlab calculation function, the image recognition processing and data analysis system of brittle materials was developed independently[4]. The main flow of the algorithm is as follows: (1) Use different scales to mesh the image. First, process the image into a $500 \times 500$ pixel size picture, then the processed image is read in and binarization is processed to store relevant date (Figure 9(a)); (2) Cover the binary image with a square box with a side length of $\delta$, calculate and count the number of square boxes $N(\delta)$ in the damaged area and save the relevant data, a set of 


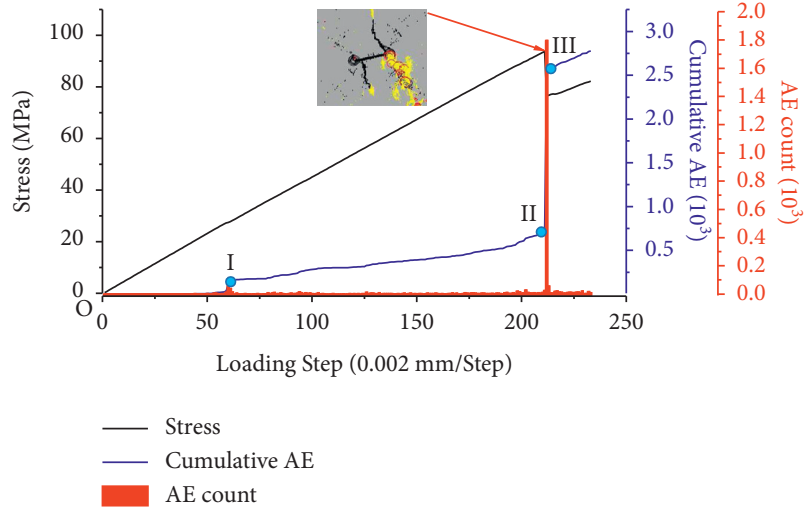

(a)

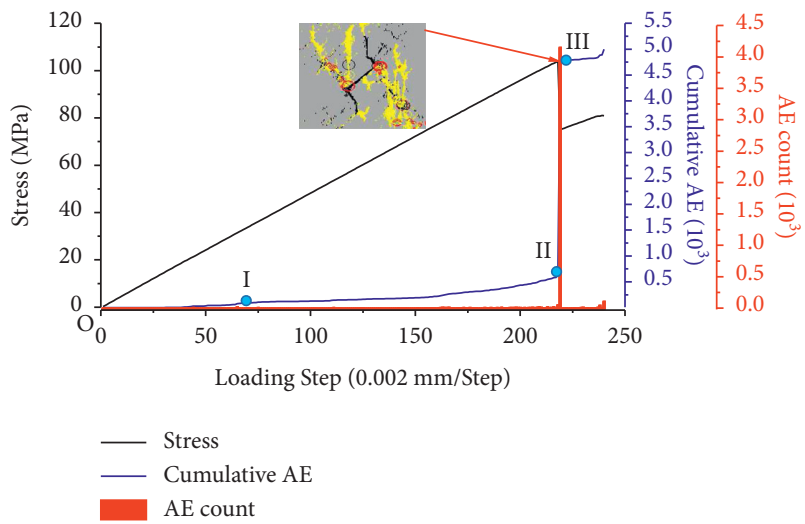

(c)

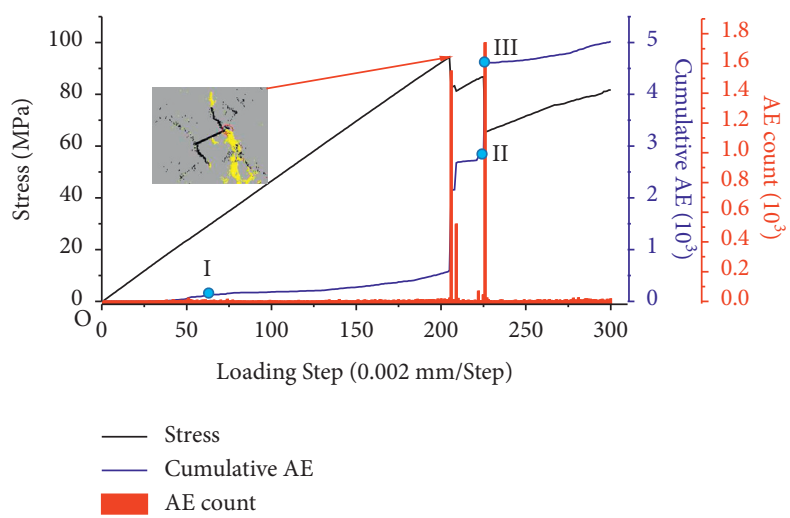

(b)

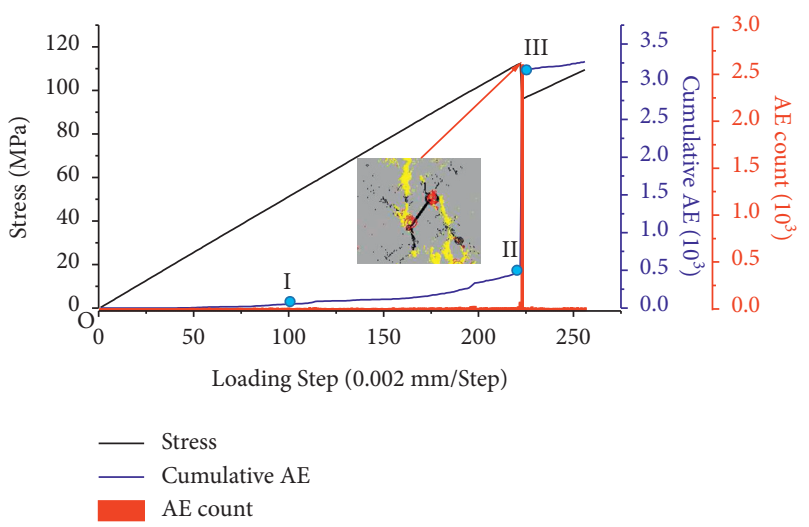

(d)

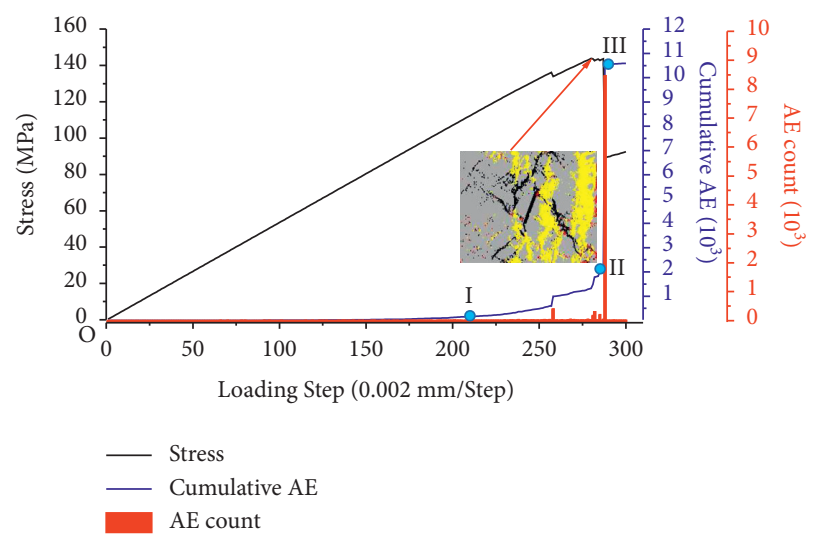

(e)

FIgURE 8: AE counts, cumulative AE counts, and stress changes with loading steps for samples with different inclination angles. (a)-(e) represents specimens $15^{\circ}, 30^{\circ}, 45^{\circ}, 60^{\circ}, 75^{\circ}$, respectively.

$N(\delta)$ and $\delta$ data could be obtained by covering the same damage area with different scales of $\delta$, and the $\delta=2^{\mathrm{n}}(\mathrm{n}=1,2,3$, ...9) as shown in Figure 9(b); (3) Take double logarithmic coordinates of $N(\delta)$ and $\delta$ for regression analysis and use the fitted line, the value of $D$ can be obtained by the slope of the fitted line.

Figure 10 depicts the process of calculating the box dimension of the acoustic emission binary image during the concrete failure process using the self-developed brittle material hole crack image recognition processing and data analysis system. When $\alpha=45^{\circ}$ and the stress level is $70 \%$,
Figure 11 shows that the fractal fitting relationship diagram of the failure of the concrete sample. Table 3 shows the acoustic emission energy values and fractal dimensions of numerical samples at various stress levels.

Under various stress settings, Figure 12 depicts the link between the sample and acoustic emission energy. Figure 12 demonstrates that as the stress level rises, the acoustic emission energy rises as well. The damage development under different stress conditions is a gradual acceleration process (increasing slope). The axial stress is near to the peak intensity when the stress level is greater than $90 \%$, which 

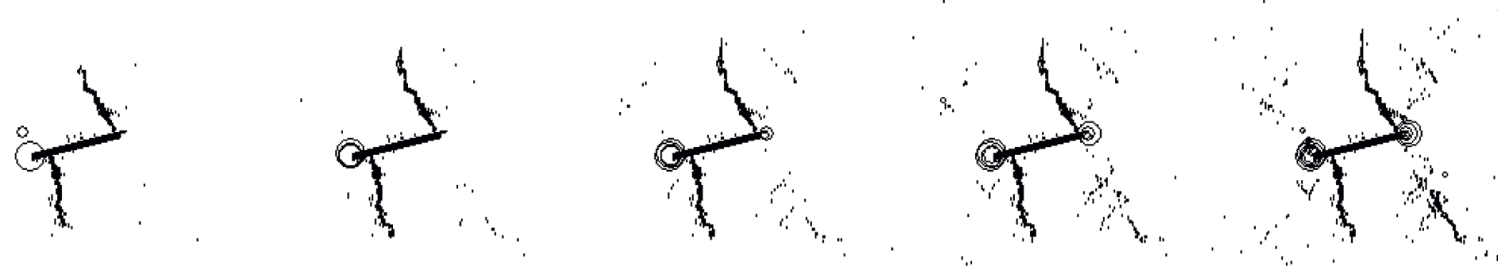

$\sigma=0.6 \sigma_{\max }$ $\sigma=0.7 \sigma_{\max }$

$\sigma=0.8 \sigma_{\text {max }}$

$\sigma=0.9 \sigma_{\max }$

$\sigma=\sigma_{\max }$

(a)

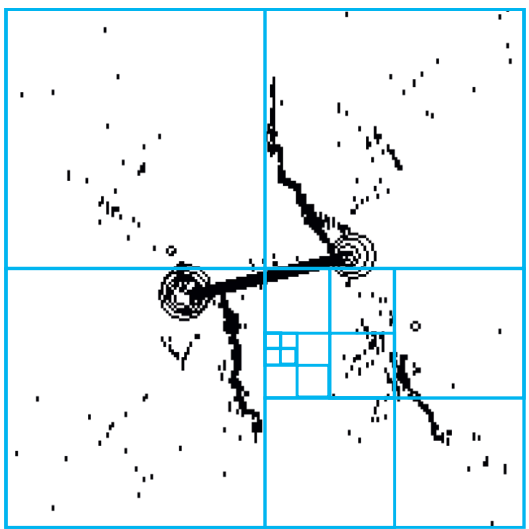

(b)

FIGURE 9: Schematic diagram of calculation of fractal dimension of concrete material fracture evolution.
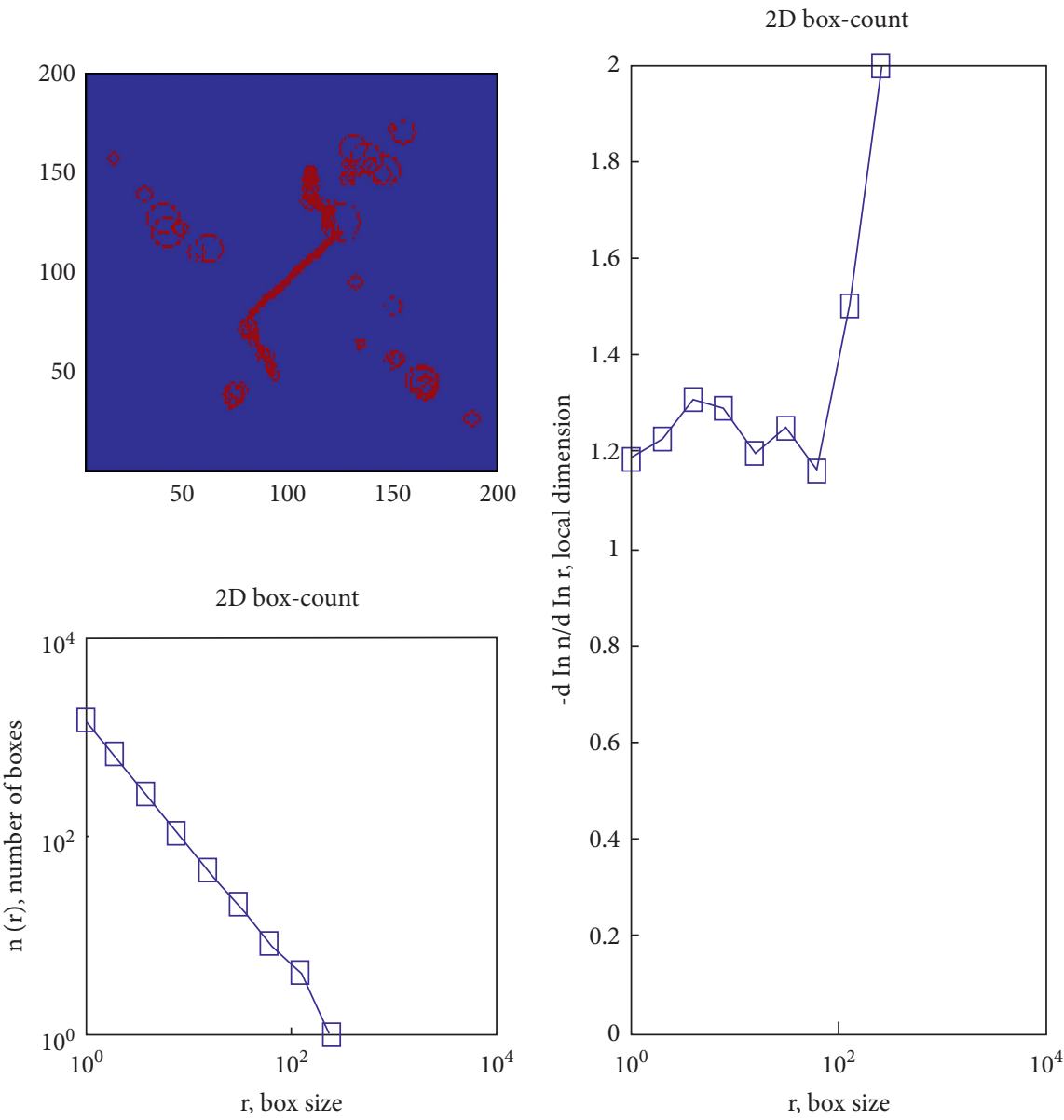

FIgURE 10: when $\alpha=45^{\circ}$, the fractal dimension is calculated (the stress level is $70 \%$ ) 


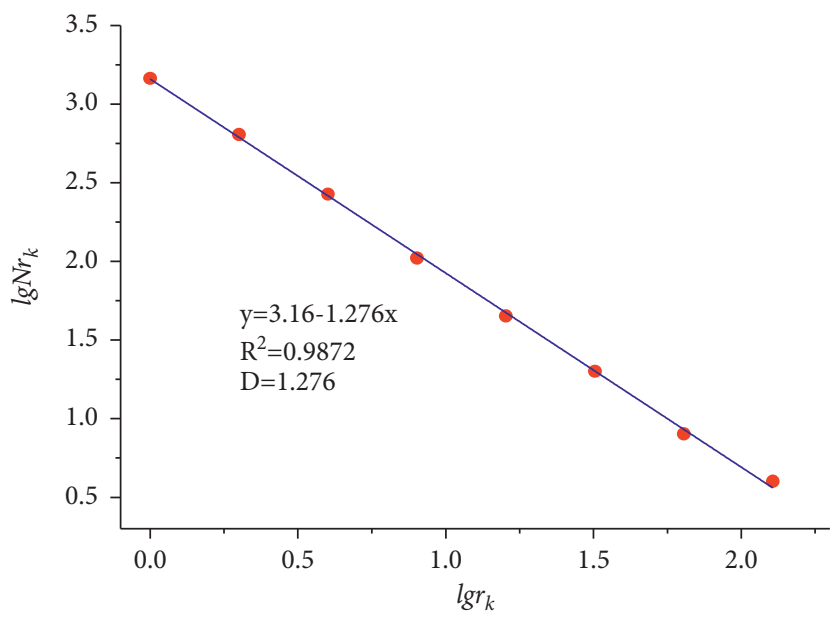

FIgURE 11: Fractal dimension of damage area calculation results when $\alpha=45^{\circ}$ (stress level of $70 \%$ ).

TABLE 3: AE energy and fractal dimension values for numerical samples at various stress levels

\begin{tabular}{|c|c|c|c|c|c|c|c|c|c|c|c|}
\hline \multicolumn{12}{|c|}{ Stress level } \\
\hline \multicolumn{2}{|l|}{ Inclination } & $10 \%$ & $20 \%$ & $30 \%$ & $40 \%$ & $50 \%$ & $60 \%$ & $70 \%$ & $80 \%$ & $90 \%$ & $100 \%$ \\
\hline \multirow[t]{3}{*}{$\alpha=15^{\circ}$} & $\mathrm{AE}$ & 54 & 73 & 159 & 206 & 295 & 314 & 382 & 434 & 519 & 717 \\
\hline & $\mathrm{D}$ & 0.840 & 0.915 & 1.073 & 1.163 & 1.198 & 1.219 & 1.242 & 1.275 & 1.34 & 1.419 \\
\hline & $\omega$ & 0.420 & 0.459 & 0.537 & 0.582 & 0.599 & 0.610 & 0.621 & 0.638 & 0.67 & 0.710 \\
\hline \multirow[t]{3}{*}{$\alpha=30^{\circ}$} & $\mathrm{AE}$ & 62 & 88 & 112 & 167 & 185 & 203 & 256 & 320 & 402 & 594 \\
\hline & $\mathrm{D}$ & 0.982 & 0.987 & 1.131 & 1.167 & 1.187 & 1.20 & 1.22 & 1.285 & 1.328 & 1.423 \\
\hline & $\omega$ & 0.491 & 0.494 & 0.567 & 0.584 & 0.594 & 0.60 & 0.61 & 0.643 & 0.664 & 0.712 \\
\hline \multirow[t]{3}{*}{$\alpha=45^{\circ}$} & $\mathrm{AE}$ & 64 & 71 & 78 & 120 & 131 & 168 & 195 & 294 & 400 & 633 \\
\hline & $\mathrm{D}$ & 0.899 & 0.962 & 1.042 & 1.101 & 1.18 & 1.251 & 1.276 & 1.303 & 1.352 & 1.44 \\
\hline & $\omega$ & 0.449 & 0.481 & 0.521 & 0.551 & 0.59 & 0.626 & 0.638 & 0.652 & 0.676 & 0.72 \\
\hline \multirow[t]{3}{*}{$\alpha=60^{\circ}$} & $\mathrm{AE}$ & 47 & 69 & 76 & 81 & 92 & 108 & 116 & 174 & 334 & 542 \\
\hline & $\mathrm{D}$ & 0.807 & 0.938 & 1.01 & 1.176 & 1.196 & 1.214 & 1.273 & 1.298 & 1.326 & 1.401 \\
\hline & $\omega$ & 0.404 & 0.469 & 0.505 & 0.588 & 0.598 & 0.607 & 0.637 & 0.649 & 0.663 & 0.701 \\
\hline \multirow[t]{3}{*}{$\alpha=75^{\circ}$} & $\mathrm{AE}$ & 44 & 83 & 96 & 119 & 141 & 144 & 172 & 180 & 421 & 1319 \\
\hline & $\mathrm{D}$ & 0.815 & 0.982 & 0.995 & 0.998 & 1.071 & 1.122 & 1.129 & 1.259 & 1.396 & 1.544 \\
\hline & $\omega$ & 0.408 & 0.491 & 0.498 & 0.499 & 0.536 & 0.561 & 0.565 & 0.630 & 0.698 & 0.772 \\
\hline
\end{tabular}

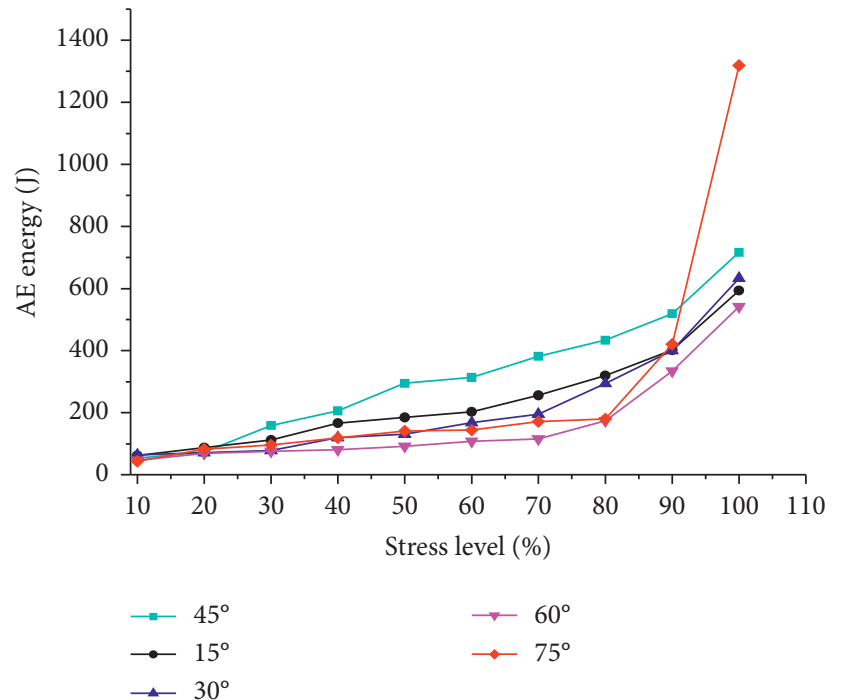

Figure 12: The connection between AE energy and various levels of stress. causing the crack to penetrate and release a large amount of energy, resulting in a large number of elements damaged, the number of acoustic energy increased sharply and the location was highly concentrated. A macroscopic failure zone (Figure 7) is formed. The acoustic emission energy value released by the sample with $\alpha=75^{\circ}$ is the greatest when the stress reaches its peak intensity. Combined with the acoustic emission evolution in Figure 7, it can be seen that the internal damage is the most serious and the macroscopic failure is the most severe.

The fractal dimension of each selected binary picture is extracted after performing fractal analysis on the corresponding damage evolution binary picture under different stress levels, and the change in dimension is shown in Figure 13. The degree of damage to the microstructure of each sample's material increases as the stress level increases, there are more and more failure elements on the corresponding damage evolution binary diagram (Figure 9(a)) and the fractal dimension increases accordingly. Meanwhile, the corresponding damage evolution binary diagram has 


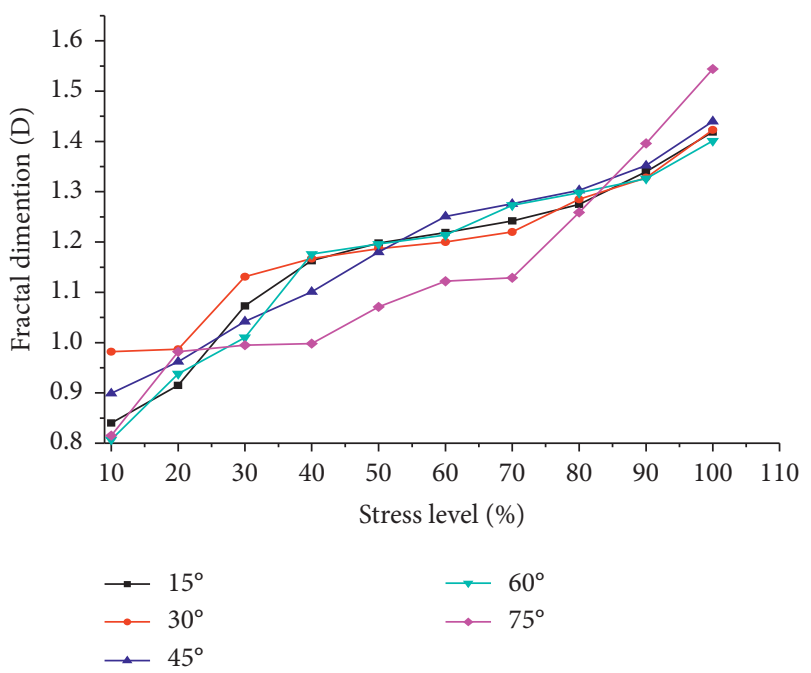

FIGURE 13: Relationship between fractal dimension and different stress levels.

more and more rupture elements (Figure 9(a)) and the fractal dimension increases. When the stress reaches the critical value, the stress suddenly drops at the same time accompanied by abrupt behaviors such as the rapid increase of failure elements and the increase of damage fractal dimension. The failure probability and fractal dimension change little when the stress level is less than $30 \%$. Material failure is actually a self-organizing process from disordered distribution to concentrated order, the failure elements develop from overall random distribution to local concentration, macroscopically it is the initiation and expansion of microcracks, the size of the fractal dimension reflects the damage. This means that there are more and more fine defects, which tend to diffuse into the entire sample. As the stress level increases, the development of damage inside the material is a gradual acceleration process, reflecting the accelerated deterioration of the mechanical properties of the material under the action of stress until it is destroyed. When Figures 11 and 12 are compared, it is clear that the evolution process of the material's internal fracture shows a general trend of increasing dimensionality. When the stress level is $100 \%$, there is a good corresponding relationship between the change of fractal dimension and the energy release, which is expressed as acoustic emission energy, the greater the release, the greater the fractal dimension, and the more serious the internal damage of the material.

3.4.2. Damage variable based on fractal theory. Although there are many methods to determine rock damage variables, most of them are still describing the quantitative relationship of micro-fracture evolution, the spatial distribution characteristics of micro-fractures have not been reflected, and the damage characteristics of rock materials cannot be truly reflected. Therefore, exploring a method that can truly reflect the process of mechanics and can be easily obtained and objectively measured is an important issue that needs to be solved urgently in the geotechnical engineering community. However, the damage and failure process of materials is actually the development, inoculation and evolution process of internal micro-fractures. Since the method of stiffness degradation is used to deal with the failure elements in the numerical model, each failure element can be regarded as a micro-fracture. Larger-scale cracks are formed by the failure elements connections. Therefore, the number and location distribution characteristics of the failure elements reflect the damage degree of the concrete specimens.

Based on the research foundation of section 3.4.1, this article attempts to establish the relative relationship between the damage variable and the damage degree represented by the box dimension, and use a more convenient quantitative method to express complex objects that are difficult to quantify. The definition of the damage variable expressed by the box dimension is as follows:

$$
\omega=\frac{D_{i}-D_{0}}{D_{\max }-D_{0}},
$$

where $\omega$ represents the damage variable, $D_{i}$ represents the fractal dimension of the internal fracture area of the material when the stress level is i\%; when the material is not loaded, $D_{o}$ denotes the fractal dimension of the internal fracture region of the material; $D_{\max }$ represents the fractal dimension of the fracture area when the material is completely failure. Since this research is a two-dimensional problem, $D_{\max }=2$. According to formula (3), the damage variable $\omega$ of the sample under different stress levels can be calculated, as shown in Table 3.

When comparing Figures. 13 and 14, it's clear that the damage variable $\omega$ and the fractal dimension $D$ have a synchronized changing trend, and both increase continuously with the increase of stress. This is due to the continuous development of a large number of micro-defects in the material in the process of deformation, and the occurrence of macroscopic failure is caused by the continuous accumulation of internal damage of the material. As the increase of the stress level, the increase of the internal failure elements of the material leads to the fuller the development and expansion of the micro-defects, the larger the damage variable $(\omega)$ and the fractal dimension. The generation and evolution of the internal fracture of the material can be described by the change. Thence, based on the damage variable $(\omega)$ of fractal theory, the damage of rock-like materials is characterized. Different crack propagation paths result from the distribution form of a single crack in concrete. Due to microscopic non-uniformity, cracks begin to form and propagate over the weak surface, which leads to the expansion of the crack along the weak surface to form an irregular propagation path, which is expressed as the fractal dimension $\mathrm{D}$ and the damage variable $\omega$ have a certain difference in the damage evolution process of the material, that is, $\omega_{75^{\circ}}>\omega_{45^{\circ}}>\omega_{30^{\circ}}>\omega_{15^{\circ}}>\omega_{60^{\circ}}$.

3.5. Application prospect analysis of damage variable based on fractal theory in damage evolution of roadway in Lannigou Gold Mine. The Lannigou Gold Mine is the largest fault- 


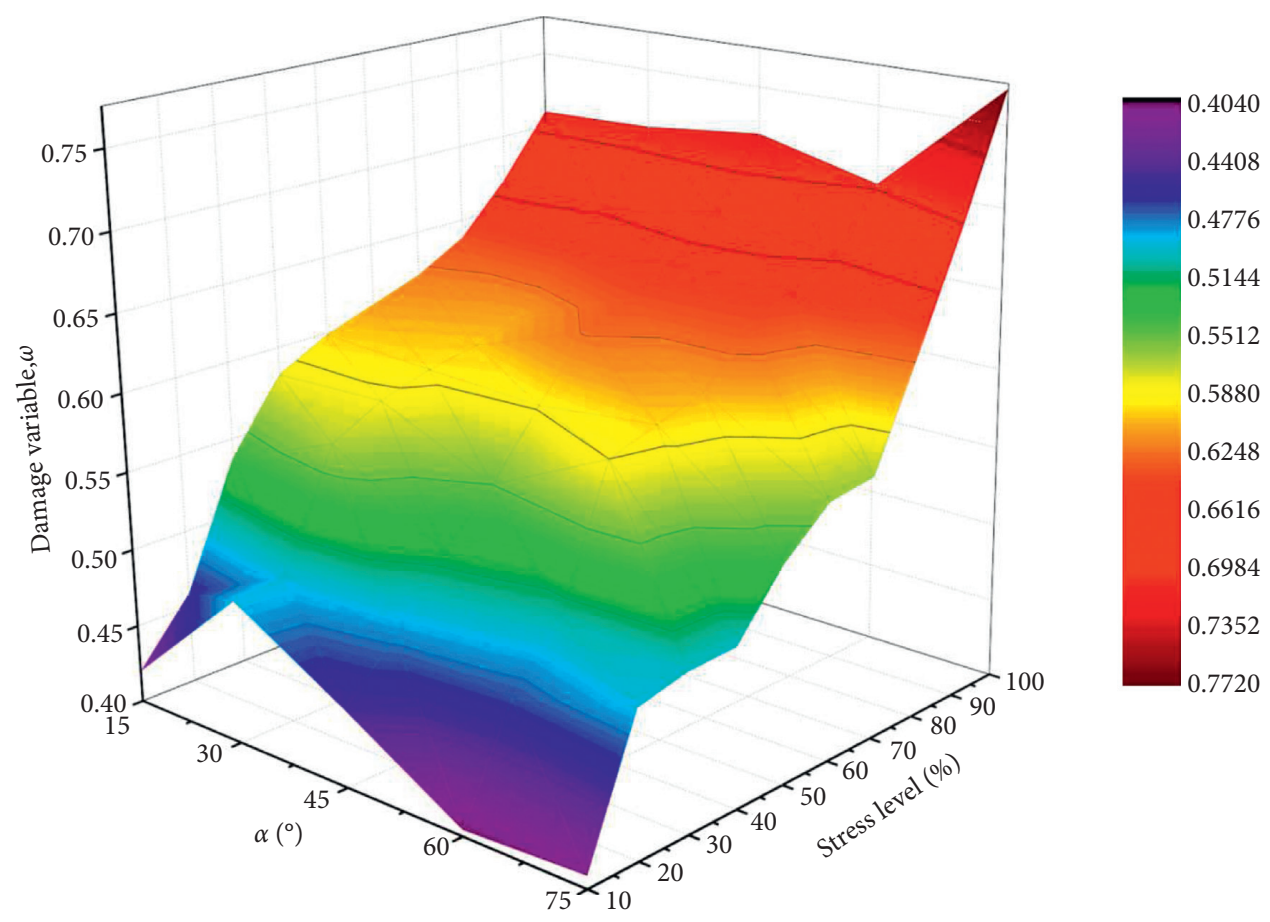

FIgURE 14: Relationship between damage variable, stress levels, and inclination.

controlled Carlin-type gold deposit in southwestern Guizhou. From 1987 to the end of 2005, the identified resources amounted to 126.25 tons, reaching an extremely large scale [29]. Multiple periods of tectonic movement have occurred in the mine, resulting in complex geological circumstances for the ore body's occurrence. During the deep mining process, the surrounding rock of the deep roadway has encountered challenges such as large deformation and support difficulties [30, 31]. The practice of deep mining engineering has been ahead of the systematic exploration of related basic theories, and there lindness, inefficiency and uncertainty in engineering practice to a certain extent. Therefore, this research can provide certain theoretical support for the optimization of deep wet shotcrete support strength and parameters of Lannigou Gold Mine.

At present, domestic and foreign researchers have obtained many research results on the damage evolution of surrounding rock in deep mining tunnels. Zhu et al. used RFPA software and assumed that the elastic modulus and strength indexes of the rock obey the Weibull distribution, and numerically simulated the initiation, expansion and penetration process of the underground surrounding rock fractures [32]. Zhang et al. aimed at the difficulty of controlling the stability of the surrounding rock in deep broken soft rock roadways, analyzed the deformation and failure characteristics and failure mechanism of the surrounding rock [33]. Dou et al. investigated the effect of shear displacement in the roadway's surrounding rock on fluid flow and solute transport in rough fractures [34]. Gao et al. studied the drilling behavior and mechanism of cores below $4500 \mathrm{~m}$ in Well Songke 2 in order to learn more about the damage mechanisms of the surrounding rocks [35].The above research has important reference value for the understanding of roadway failure mechanism, but there are few reports on how to quantify the degree of roadway failure. However, the essence of roadway failure is the macro-mechanical response generated by the accumulation of internal damage to a certain extent. If the whole process of roadway from damage to final failure can be quantitatively characterized, it will help to reveal the damage of the surrounding rock of deep roadway and provide a reference for the stability control of the surrounding rock of the roadway.

This study describes the damage evolution process inside the material based on fractal theory, and proposes a damage variable index $(\omega)$ based on fractal theory. Using $\omega$ as a parameter to characterize the degree of material damage, the macroscopic failure characteristics of the material and its internal fracture evolution are unified and the inconvenience of too many mechanical parameters involved in the damage definition is overcome, and provides a new method for quantitatively studying the damage evolution characteristics of rock-like materials. Based on the study in this article, we independently developed a system for image recognition and processing of pores and cracks in brittle materials and data analysis. In view of the control problems of the surrounding rock of the Lannigou gold mine and the study ideas in this article, this system can calculate the fractal dimension to analyze the damage evolution of the roadway's surrounding rock. In order to better understand the damage evolution process of the surrounding rock of the roadway, a damage variable index based on the fractal dimension is presented to measure the degree of growth of cracks in the surrounding rock, so as to provide certain guidance for the safe production of the mine. 


\section{Conclusion}

(1) The box dimension is used to describe the internal damage evolution binary diagram of the material, and it is found that the damage fractal dimension has a linear relationship with the stress at different stages. A damage variable index $(\omega)$ based on the fractal theory is proposed, and $\omega$ is used as a parameter to characterize the degree of material damage. The macroscopic failure characteristics of the material and its internal fracture evolution are unified and the inconvenience of too many mechanical parameters involved in the damage definition is overcome. The larger the $\omega$, the higher the degree of damage to the material. The proposed index introduces a new way for studying the damage evolution characteristics of rock-like materials in a quantitative manner.

(2) The crack inclination angle has a substantial impact on the elastic modulus and compressive strength of concrete materials, and exhibits non-linear properties as the angle increases. The material's interior microscopic non-uniformity causes an uneven internal stress distribution, and wing crack initiation and propagation paths are somewhat random. As the prefabricated crack inclination angle increases, the wing crack length shortens, and the wing crack initiation point moves closer to the prefabricated crack tip.

(3) Due to the influence of the internal non-uniformity of the material, when $15^{\circ} \leq \alpha \leq 45^{\circ}$, the wing crack initiation and expansion is easier. When $\alpha=60^{\circ}$ and $\alpha=75^{\circ}$, it is difficult for the wing crack to initiate and expand. When approaching the peak stress, the crack initiation and spread suddenly. Finally, the splitting failure is formed.

(4) The crack specimens with different inclination angles have similar evolution characteristics of acoustic emission. The AE energy is small in the early stage of loading, and it increases rapidly when the stress is close to the peak. The cumulative AE energy reflects the entire destruction process of the material under compression. It expands exponentially as the loading step is increased, the growth process can be separated into three phases: flat, acceleration, and inflation.

\section{Data Availability}

Some or all data, models, or code generated or used during the study are available from the corresponding author by request.

\section{Conflicts of Interest}

The authors declares that there is no conflict of interest regarding the publication of this paper.

\section{Acknowledgments}

The study was funded by the Guizhou Province Science and Technology Support Program Project (No: QIANKEHE
Support [2021] General 516); National Natural Science Foundation of China (No: 51964007, 41962008, 51774101).

\section{References}

[1] C. Zhu, M.-c. He, B. Jiang, X.-z. Qin, Q. Yin, and Y. Zhou, "Numerical investigation on the fatigue failure characteristics of water-bearing sandstone under cyclic loading," Journal of Mountain Science, vol. 18, no. 12, pp. 3348-3365, 2021.

[2] G. C. Shi, X. J. Yang, H. C. Yu, and C. Zhu, "Acoustic emission characteristics of creep fracture evolution in double-fracture fine sandstone under uniaxial compression," Engineering Fracture Mechanics, vol. 210, pp. 13-28, 2018.

[3] C. Zhu, M. C. He, X. H. Zhang, Z.-g Tao, Q. Yin, and L.-f Li, "Nonlinear mechanical model of constant resistance and large deformation bolt and influence parameters analysis of constant resistance behavior," Rock and Soil Mechanics, vol. 42, no. 7, pp. 1911-1924, 2021.

[4] H. Liu, Y. Zuo, Z. Wu et al., "Fractal analysis of mesoscale failure evolution and microstructure characterization for sandstone using DIP, SEM-EDS, and micro-CT," International Journal of Geomechanics, vol. 21, no. 9, Article ID 04021153, 2021.

[5] Z. Li, L. Li, B. Huang et al., "Numerical investigation on the propagation behavior of hydraulic fractures in shale reservoir based on the DIP technique," Journal of Petroleum Science and Engineering, vol. 154, pp. 302-314, 2017.

[6] C. Cao, W. Zhang, J. Chen, B. Shan, S. Song, and J. Zhan, "Quantitative estimation of debris flow source materials by integrating multi-source data: a case study," Engineering Geology, vol. 291, Article ID 106222, 2021.

[7] R. C. Nolen-Hoeksema and R. B. Gordon, "Optical detection of crack patterns in the opening-mode fracture of marble," International Journal of Rock Mechanics and Mining Sciences, vol. 24, no. 4, pp. 135-144, 1987.

[8] S.-Q. Yang and H.-W. Jing, "Strength failure and crack coalescence behavior of brittle sandstone samples containing a single fissure under uniaxial compression," International Journal of Fracture, vol. 168, no. 2, pp. 227-250, 2011.

[9] C. H. Park and A. Bobet, "Crack coalescence in specimens with open and closed flaws: a comparison," International Journal of Rock Mechanics and Mining Sciences, vol. 46, no. 5, pp. 819-829, 2009.

[10] A. Manouchehrian, M. Sharifzadeh, M. F. Marji, and J. Gholamnejad, "A bonded particle model for analysis of the flaw orientation effect on crack propagation mechanism in brittle materials under compression," Archives of civil and mechanical engineering, vol. 14, no. 1, pp. 40-52, 2014.

[11] X. P. Zhang and L. N. Y. Wong, "Cracking processes in rocklike material containing a single flaw under uniaxial compression: a numerical study based on parallel bonded-particle model approach," Rock Mechanics and Rock Engineering, vol. 45, no. 5, pp. 711-737, 2012.

[12] T. Liu, B. Lin, W. Yang, Q. Zou, J. Kong, and F. Yan, "Cracking process and stress field evolution in specimen containing combined flaw under uniaxial compression," Rock Mechanics and Rock Engineering, vol. 49, no. 8, pp. 3095-3113, 2016.

[13] R. C. Xu, "Influence of flaw inclination angle on cracking behavior of rock-like materials under uniaxial compression," Advances in Materials Science and Engineering, vol. 2019, no. 1, 10 pages, Article ID 6942586, 2019.

[14] L. M. Taylor, E.-P. Chen, and J. S. Kuszmaul, "Microcrackinduced damage accumulation in brittle rock under dynamic 
loading," Computer Methods in Applied Mechanics and Engineering, vol. 55, no. 3, pp. 301-320, 1986.

[15] T. J. Ahrens and A. M. Rubin, "Impact-induced tensional failure in rock," Journal of Geophysical Research: Planets, vol. 98, no. E1, pp. 1185-1203, 1993.

[16] H. Xie, Fractals in Rock Mechanics, A A Balkema, Rotterdam, Netherlands, 1993.

[17] M. Gao, J. Xie, Y. Gao et al., "Mechanical behavior of coal under different mining rates: a case study from laboratory experiments to field testing," International Journal of Mining Science and Technology, vol. 31, no. 5, pp. 825-841, 2021.

[18] H. Liu, L. Zheng, Y. Zuo et al., "Study on mesoscopic damage evolution characteristics of single joint sandstone based on micro-CT image and fractal theory," Shock and Vibration, vol. 2021, Article ID 6547028, 18 pages, 2021.

[19] Q. Yu, W. Zhu, C. a. Tang, and T. Yang, "Impact of rock microstructures on failure processes - numerical study based on DIP technique," Geomechanics and Engineering, vol. 7, no. 4, pp. 375-401, 2014.

[20] C. Tang, "Numerical simulation of progressive rock failure and associated seismicity," International Journal of Rock Mechanics and Mining Sciences, vol. 34, no. 2, pp. 249-261, 1997.

[21] Q. L. Yu, Digital Image Processing-Based Numerical Methods for Failure Process Analysis of Rock-like materials, Northeastern University, Shenyang, China, 2008.

[22] W. Weibull, "A statistical distribution function of wide applicability," Journal of Applied Mechanics, vol. 18, no. 3, pp. 293-297, 1951.

[23] F. Rossi, A. Di Carlo, and P. Lugli, "Microscopic theory of quantum-transport phenomena in mesoscopic systems: a Monte Carlo approach," Physical Review Letters, vol. 80, no. 15 , pp. $3348-3351,1998$.

[24] R. Y. Rubinstein and D. P. Kroese, Simulation and the Monte Carlo Method. 707, John Wiley \& Sons, Hoboken, NJ, USA, 2011.

[25] B. H. G. Brady and E. T. Brown, Rock Mechanics for Underground mining, pp. 106-108, Chapama and Hall, London, UK, 1993.

[26] D. Li, E. Wang, X. Kong, M. Ali, and D. Wang, "Mechanical behaviors and acoustic emission fractal characteristics of coal specimens with a pre-existing flaw of various inclinations under uniaxial compression," International Journal of Rock Mechanics and Mining Sciences, vol. 116, pp. 38-51, 2019.

[27] A. Bobet, "The initiation of secondary cracks in compression," Engineering Fracture Mechanics, vol. 66, no. 2, pp. 187-219, 2000.

[28] J. Jin, P. Cao, and C. Z. Pu, "Effect of preformed fracture geometry parameters on failure mode and strength of rocklike materials," Journal of Central South University, vol. 45, no. 2, pp. 529-535, 2014, in Chinese.

[29] C. Maohong, M. Jingwen, F. P. Bierlein, T. Norman, and P. J. Uttley, "Structural features and metallogenesis of the carlin-type Jinfeng (Lannigou) gold deposit, Guizhou Province, China," Ore Geology Reviews, vol. 43, no. 1, pp. 217-234, 2011.

[30] L. Zheng, Y. Zuo, and Y. Hu, "Deformation mechanism and support technology of deep and high-stress soft rock roadway," Advances in Civil Engineering, vol. 2021, Article ID 6634299, 14 pages, 2021.

[31] D. Chen, H. Chen, and W. Zhang, "An analytical solution of equivalent elastic modulus considering confining stress and its variables sensitivity analysis for fractured rock masses,"
Journal of Rock Mechanics and Geotechnical Engineering, 2021.

[32] W. C. Zhu, J. Liu, C. A. Tang, X. D. Zhao, and B. H. Brady, "Simulation of progressive fracturing processes around underground excavations under biaxial compression," Tunnelling and Underground Space Technology, vol. 20, no. 3, pp. 231-247, 2005.

[33] G. C. Zhang and F. L. He, "Deformation failure mechanism of high stress deep soft roadway and its control," Journal of Mining \& Safety Engineering, vol. 32, no. 4, pp. 571-577, 2015.

[34] Z. Dou, S. X. Tang, and X. Y. Zhang, "Influence of shear displacement on fluid flow and solute transport in a 3D rough fracture," Lithosphere, vol. 2021, Article ID 1569736, 2021.

[35] M. Z. Gao, H. C. Hao, and S. N. Xue, "Discing behavior and mechanism of cores extracted from Songke-2 well at depths below 4500 m," International Journal of Rock Mechanics and Mining Sciences, vol. 149, Article ID 104976, 2022. 